\title{
The use of rapid prototyping in undergraduate design education
}

\author{
R. O. Buchal, D. Phillips \\ Faculty of Engineering \\ The University of Western Ontario
}

\begin{abstract}
The building and testing of physical prototypes has always been a key phase of the engineering design process. Often, students rush to the prototype stage with insufficient modeling and analysis. As a result, the process resembles "trial and error" more than systematic engineering design. Furthermore, engineering schools lack the facilities and students lack the skills to construct more than very crude prototypes with little resemblance to the $C A D$ models or to the final design. On the other hand, engineering schools typically have state-of-the-art CAD software. As an alternative to physical prototyping, the emphasis is shifting to "virtual" prototyping using CAD models and simulation. Many design attributes like appearance, performance, etc. can be established through simulation with a high degree of reliability. Furthermore, the recent availability of low cost rapid prototyping technology makes it possible to quickly and easily produce physical parts directly from the CAD model.
\end{abstract}

The University of Western Ontario Faculty of Engineering has recently established a rapid prototyping facility for undergraduate design projects. The facility is available to students from all programs and years. The facility is professionally managed by technicians from University Machine Services (UMS). Several rapid prototyping technologies are available, including Fused Deposition Modelling (Stratasys FDM 3000 and Stratasys Vantage SE) and $3 D$ printing (Z-Corp Z510 3D Printer). The Z-Corp 3D printer is capable of processing a batch of parts with a total volume of 1120 cubic inches in 20 hours, at a cost in materials of under $\$ 5$ per cubic inch. The Z-Corp printer has a resolution of 600 dpi and 256 colours, and is capable of accurately reproducing all the colours on a CAD model including texture maps. To have a part made, students simply save their CAD model as a VRML file, and submit the file for processing. Jobs are batched, and the machine is setup and run by UMS personnel. Some finishing work is completed by the students. The anticipated turnaround time is a day or two, and the typical prototype cost is under \$50. These services became available in January 2006. The final paper will include experiences gained over the coming weeks. 